\title{
Multifractal-based assessment of Gilsocarbon graphite microstructures
}

Yelena Vertyagina* and Thomas James Marrow

University of Oxford, Parks Road, Oxford OX1 3PH, UK

\begin{abstract}
Three-dimensional X-ray tomographs of the microstructures of virgin and radiolytically oxidised polygranular Gilsocarbon nuclear graphite have been analysed with a multifractal approach, to consider the evolution of the porous structures of the filler particles and matrix regions. Correlations between the Rényi dimensions and structural parameters such as the total porosity, elastic modulus, and pore sizes have been revealed. The multifractal analysis has been used to develop an algorithm to detect the spatial distribution of filler particles, which has been applied to analyse the effects of filler particles on a crack path.
\end{abstract}

\section{Introduction}

Self-similar and self-affine sets called fractals and multifractals [1, 2] can be found in many microstructures. Fractal and multifractal analysis can describe numerically the evolution of complex systems and be used to recognise the mechanisms of the processes that occur in such systems and affect their behaviour. There are many examples where empirical connections have been established between fractal parameters and microstructural characteristics that can be experimentally defined. For instance, a study of pore space heterogeneity in carbonate rocks [3] showed that pore structures and the solid component distribution within the material represented a multifractal system. The relationship between fractal dimensions and pore spaces has also been considered in other porous media [4], and the multifractal spectrum of pore structure has been correlated with the material's permeability in geological chalk [5], sedimentary rocks [6], and rock structures [7]. The multifractal parameters find a relationship with the degree of cement hydration in cement paste [8], and multifractal behaviour of corroded steel surfaces has been observed also [9]. The fractal dimension of the surface topography was used as a parameter for evaluation of the fatigue damage in Al-alloy

*Corresponding author. Tel.: 075 80225833. E-mail: yelena.vertyagina@materials.ox.ac.uk (Yelena Vertyagina) 
specimens [10], as a measure of the specific energy dissipated in the deformed austenite steel [11], and as a parameter that characterised the type of fracture in polymer-matrix composites [12]. A relationship between the multifractal parameters and the crack pattern evolution is noted in [13], and fractal analysis can also been involved in pattern recognition algorithms [14].

Polygranular nuclear graphite is an interesting porous material with current technological importance in the UK, and it has potential applications in some Generation IV high temperature advanced nuclear fission reactors [15]. Gilsocarbon graphite is a near-isotropic medium grained graphite grade that is used as a neutron moderator in the UK advanced gascooled nuclear reactors (AGR), where it experiences fast neutron irradiation and radiolytical oxidation. Graphite for nuclear applications is typically manufactured from calcined cokes, re-impregnated with coal or petroleum pitches, so that after graphitization at temperatures in excess of $2500{ }^{\circ} \mathrm{C}$ the resulting heterogeneous microstructure comprises filler particles within a matrix of ground filler "flour" and graphitized pitch. The overall material contains many pores, and also flaws arising from manufacture [16].

Oxidation changes the graphite's porous microstructure, and increased porosity can lead to a significant decrease in its mechanical properties of elastic modulus and strength [17-19]. There is a need to develop advanced methods to quantify such changes in graphite structure and to relate this to properties, and some studies [20] have considered the fractal features of graphite porosity. A recent fractal analysis of 2D optical micrographs of a non-specified porous graphite observed heterogeneity and measured the fractal nature at different scales [21]. A quite comprehensive study of fractal morphology for isotropic RID graphite (from the Research Institute Delft) and anisotropic PGA graphite (Pile Grade A, from the UK Magnox reactors) [22] used small angle neutron scattering techniques and neutron imaging to confirm the fractal properties of the microstructure over 6 orders of magnitude of length scale.

The objective of this study was to investigate the multifractal morphology of the Gilsocarbon graphite microstructure, and its response to oxidation. We show that the porosity in Gilsocarbon can be quantitatively described by multifractal parameters, which differ for the matrix and filler particles. This leads to a multifractal-based algorithm to detect the characteristic structures of filler particles, which allows their three-dimensional spatial distribution to be mapped for the first time. The mapping is applied to examine the interaction of a crack with the graphite microstructure. 


\section{Gilsocarbon graphite microstructure}

X-Ray tomography data were obtained at the UK Diamond Light Source (experiment EE8519, I12 beam line) for a sample of virgin (i.e. not irradiated nor oxidised) Gilsocarbon graphite with a size of $5.2 \times 4.6 \times 3.2 \mathrm{~mm}$ at a voxel resolution of $1.8 \mu \mathrm{m} /$ voxel. This graphite is of the same type used in the UK Heysham II AGR (referred to as HYB). A second sample of virgin graphite was of the type used in the UK Hinkley Point B (HPB) AGR; laboratory XRay tomographs were obtained with a resolution of $2.5 \mu \mathrm{m} /$ voxel for a volume size of 4.9 x 4.9 × 3.9 mm. Laboratory tomography data for 10 radiolytically oxidised HPB graphite samples were provided by EDF Energy Generation in the range of weight loss between 8\% and $68 \%$ with a resolution of $2.5 \mu \mathrm{m} /$ voxel and sizes of approximately $5 \times 5 \times 3 \mathrm{~mm}$. The radiolytically oxidised samples form part of an accelerated aging test programme [23]. Their weight losses, also provided by EDF Energy Generation, were calculated from density measurements and are relative to the average density of the virgin graphite.

The matrix and coarse filler particles of the Gilsocarbon graphite microstructure have visibly different porous structures. Filler particles are typically of ellipsoidal shapes with the pores that exhibit an onion-like structure, whilst the matrix' pores are very irregular with a high degree of interconnectivity. Example grayscale tomography slices of virgin HYB and HPB alongside with low and highly oxidised HPB samples are shown in Figure 1, with the porous microstructures of selected filler particles and matrix regions identified. The HPB graphites were manufactured by AGL (Anglo Great Lakes), whereas HYB was manufactured by UCAR (Union Carbide Carbon Company).

Filler particles and matrix sub-volumes were randomly extracted from the datasets for analysis. The average sizes of the selected particles are presented in Figure 1e. A total of 14 filler particles and 7 matrix sub-volumes were analysed for each dataset, with the exception of virgin HPB, for which only filler particle data were obtained. The pore structure in the virgin HPB matrix could not be resolved sufficiently well for characterisation, and no matrix regions were extracted. The two grades of nuclear graphite in the virgin state (i.e. at $0 \%$ weight loss) have different filler particle size distributions with the HYB particles being coarser. Consequently, the sizes of the matrix regions that could be extracted from between the filler particles are also different between the two grades; $1 \mathrm{~mm}^{3}$ for HYB graphite and $0.5^{3} \mathrm{~mm}^{3}$ for HPB graphite. 
All datasets have been segmented using a multi-step thresholding with repeating smoothing and binarisation steps for pore boundary determination and large pore filling. The observable porosities were then measured for the full microstructures. The total measured porosity value is $~ 16 \%$ for the virgin HYB microstructure, $12 \%$ for virgin HPB microstructure and ranges from 21.3-72\% for the oxidised HPB graphite. These values are lower than the real porosities, however, due to the limited resolution of the datasets. The actual total porosity of virgin graphite is around 20\% [24]. Nonetheless, the tomography data have sufficient resolution to characterise the majority of the pores. The average porosities of the sub-volumes are presented in Figure $1 \mathrm{f}$ in comparison with the full microstructures as a function of the weight loss by radiolytic oxidation. The matrix regions in oxidised HPB typically have a higher pore content that the filler particles, which tend to have low porosity when the weight loss is below 20\%, and higher porosity above this. Qualitative differences in the porosity structures of the filler and matrix are also observable from the tomographs (Figure 1). Although the size of the filler particles differs between HYB and HPB graphites, there is no qualitative difference in their visible pore structures in the virgin state. 


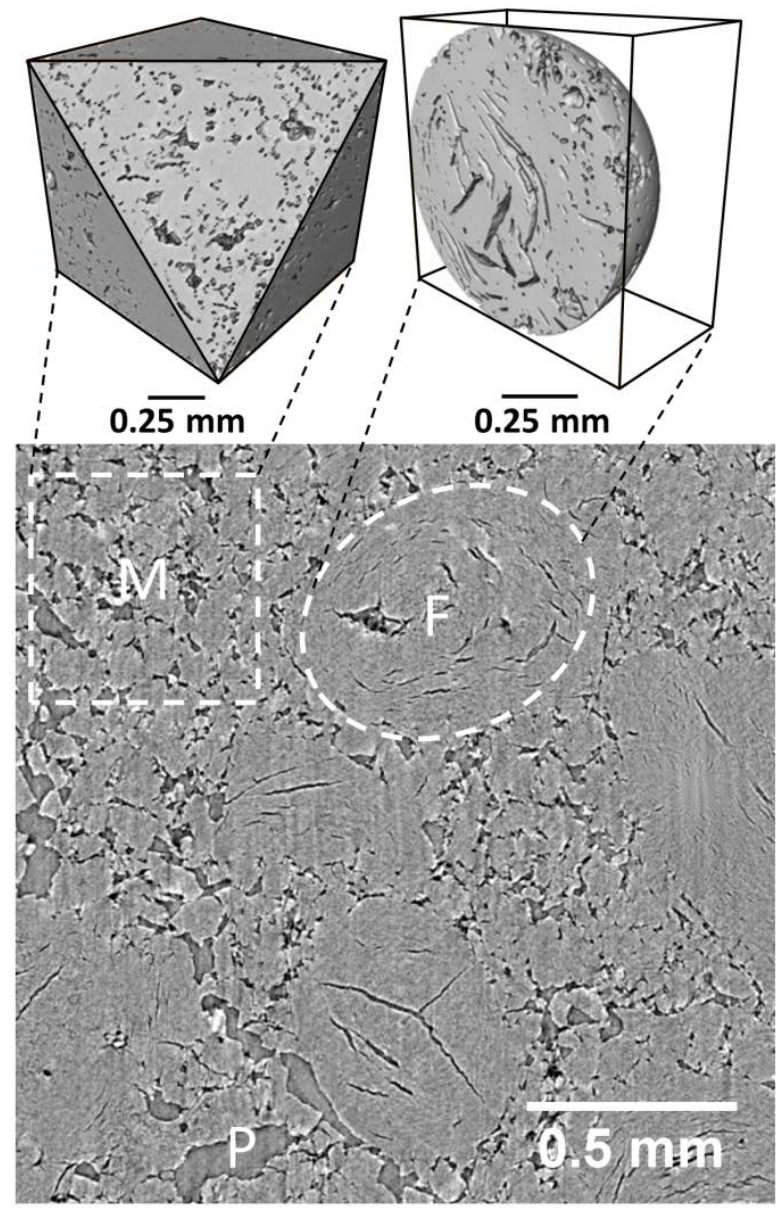

a)

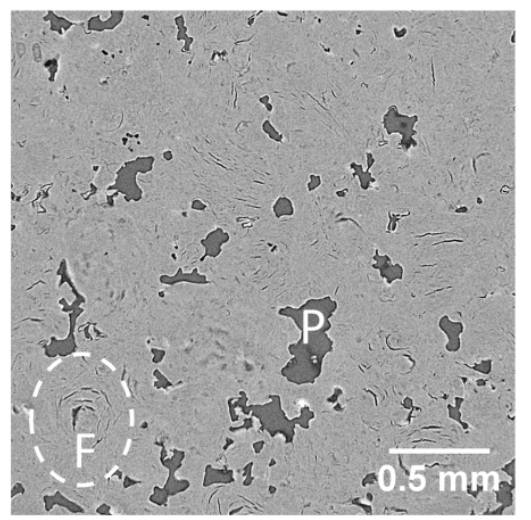

c)
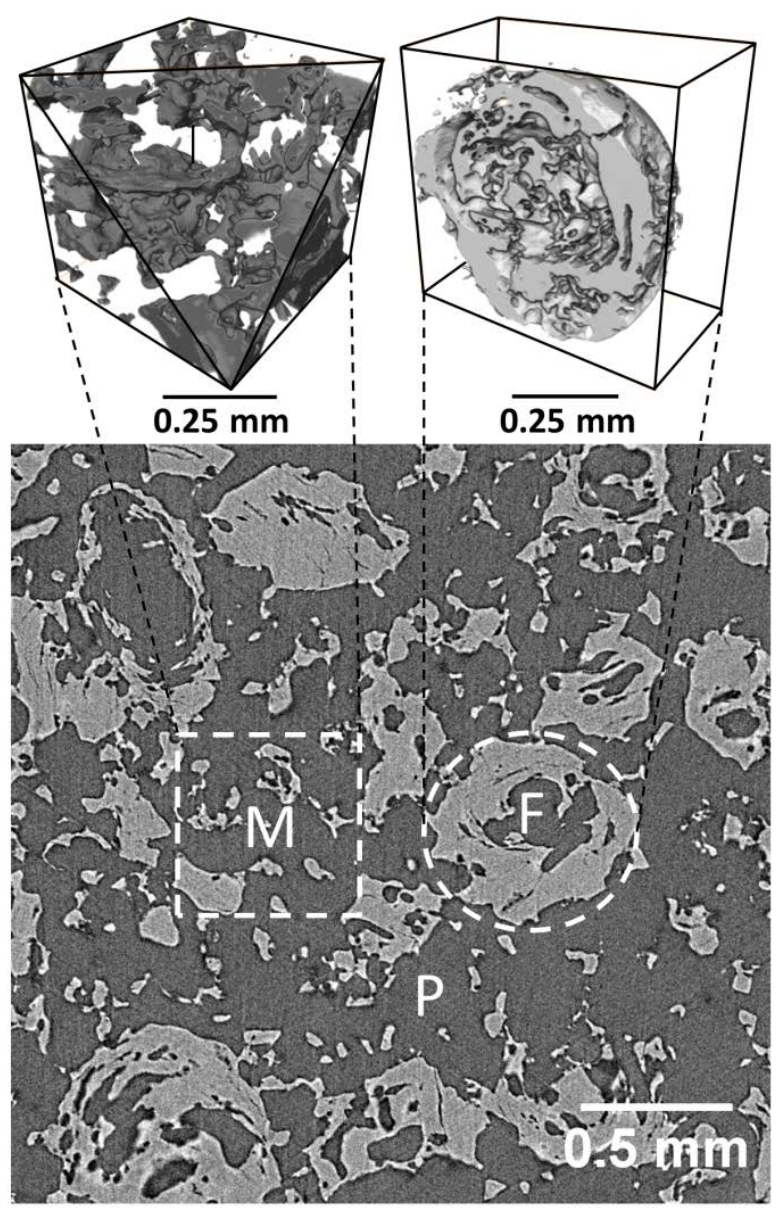

b)

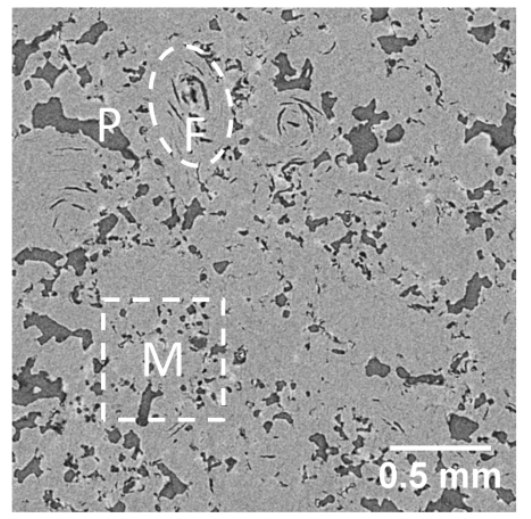

d)

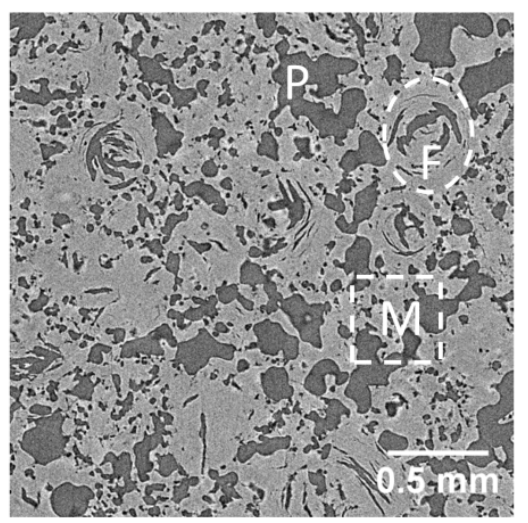

e 


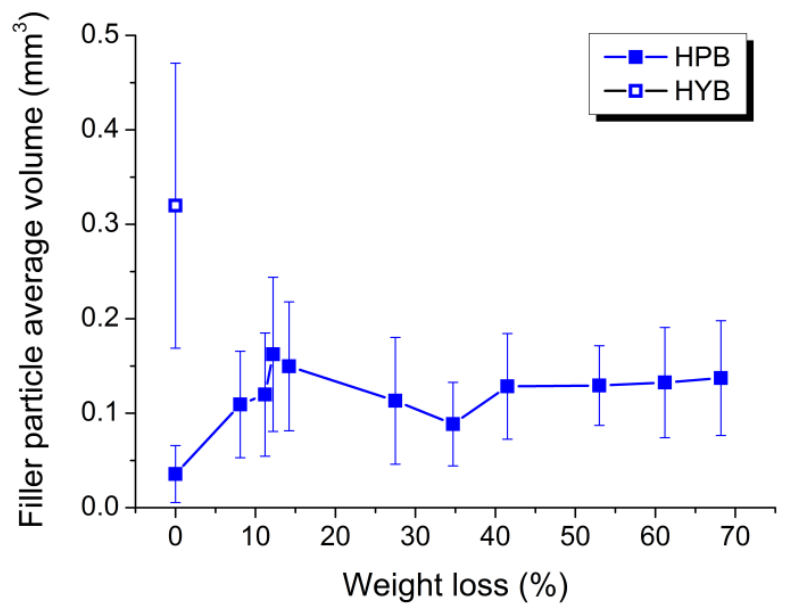

f)

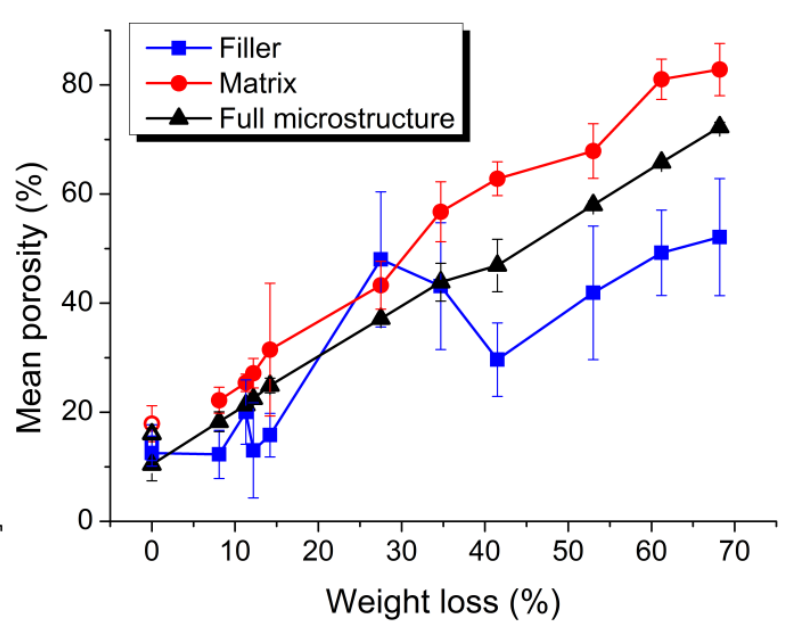

g)

Figure 1 - 2D X-Ray tomography slices of virgin HYB graphite (a), highly oxidised HPB graphite with weight loss of 68.2\% (b), virgin HPB graphite (c), low oxidised HPB graphite with weight loss of $8.1 \%$ (d), and oxidised HPB graphite with weight loss of $27.5 \%$ (e), and 3D reconstructed microstructures of the extracted filler particles (F) and matrix (M) subvolumes, pores are denoted by 'P'; f) average sizes of the extracted filler particles; g) measured porosities of the selected sub-volumes and full microstructures. Error bars correspond to standard deviation. HPB graphite is shown by solid points, HYB graphite is represented by open points.

\section{Multifractal analysis technique}

Multifractal analysis is a technique for quantitative description of the structural state of a selected dataset. A multifractal structure represents a set of fractal structures with a spectrum of fractal dimensions. This approach operates with a number of parameters such as Rényi generalised dimensions, multifractal spectrum function, statistical sum and spatial probabilities [2, 25]. As we are considering a porous structure, we use a box-counting method for an estimation of the spatial probabilities, where the binary microstructure is divided into sub-volumes so that $N$ corresponds to the number of non-empty subsets and $r$ denotes the linear size of each sub-volume. This division process is repeated further for decreasing values of $r$, and this leads to the definition of the most general Rényi generalised dimension parameter, the Hausdorff dimension: 


$$
D_{0}=-\lim _{r \rightarrow 0} \frac{\ln N(r)}{\ln r}
$$

The Hausdorff dimension characterises the degree of spatial occupation by the microstructure. This parameter lies between 2 and 3 for 3D microstructures and tends to have a lower value for clustered sets.

The spatial probability $p_{i}$ allows determination of other two generalised Rényi dimensions, $D_{1}$ and $D_{2}$. The information dimension $D_{1}$ is calculated through the Shannon information entropy $S$ [26] according to the following relationship:

$$
D_{1}=-\lim _{r \rightarrow 0} \frac{S(r)}{\ln r} ; \quad S=\sum_{i=1}^{N} p_{i} \ln p_{i}
$$

The information dimension, due to the corresponding information entropy, reflects the degree of structural organisation. A higher value of $S$ demonstrates a greater disorder in the structure, whilst a lower value may indicate a self-organisation process, which has led to structural reconfiguration. The correlation dimension $D_{2}$ can be estimated with the use of the correlation function $I$, which defines the probability of finding two structure elements within the radius $r$ :

$$
D_{2}=\lim _{r \rightarrow 0} \frac{\ln I(r)}{\ln r} ; \quad I=\sum_{i=1}^{N} p_{i}^{2}
$$

Both $D_{1}$ and $D_{2}$ are typically more sensitive to the structural changes than $D_{0}$ and their variation, therefore, might be associated with changes in physical parameters that are dependent on the microstructure state, for example density, the distribution of porosity and elastic modulus [27-29].

\section{Results}

The multifractal approach has been applied to the full volumetric data of the Gilsocarbon graphite microstructures in virgin and oxidised states as well as to the sub-volumes of the filler and matrix extracted from the original microstructures. 


\subsection{Multifractal parameters of Gilsocarbon graphite microstructure}

The multifractality of the Gilsocarbon microstructures was first checked. Computations of the Rényi dimensions $D_{0}, D_{1}$, and $D_{2}$ have been performed for the porous structure at three different resolutions: 2.5, 5, and $10 \mu \mathrm{m} / \mathrm{voxel}$, which were obtained for the same microstructure by downsizing using the 'scale' function of ImageJ [30] with bilinear interpolation and averaging. Example data for the virgin HYB microstructure are shown in Figure 2; here the total size of the examined dataset was $3 \times 3 \times 3 \mathrm{~mm}$ and the size of the selected filler particles and matrix sub-volumes did not exceed 1x1x1 mm. A scaling function has been found in all cases. This means that each of three dimensions, defined by the slope of the corresponding line, is conserved at the considered length scale. This gives us an opportunity to operate with the quite large volumes with low resolutions, the size of which would be restricted by the computational performance only.

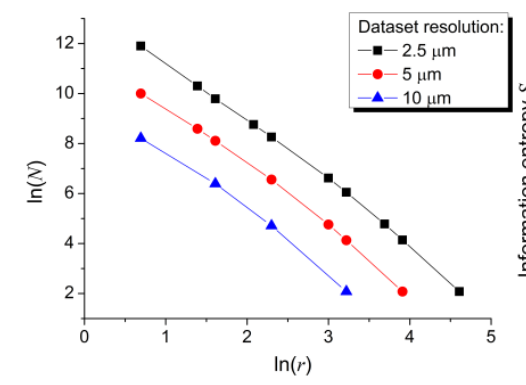

a)

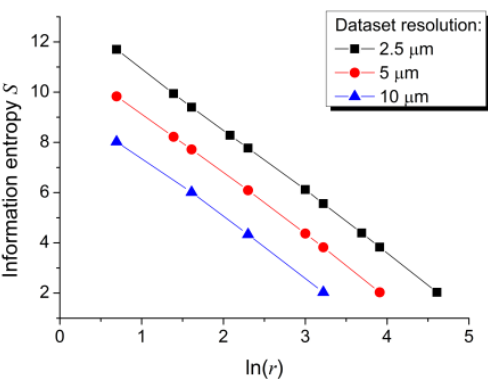

b)

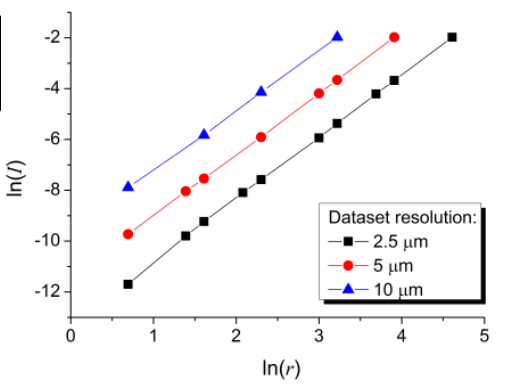

c)

Figure 2 - Scaling of the fractal parameters for virgin HYB graphite microstructure: a) Hausdorff dimension $D_{0}$; b) information dimension $D_{1}$; c) correlation dimension $D_{2}$. All dimensions are calculated as the slopes of the corresponding lines.

The Hausdorff dimension, $D_{0}$, is presented as a function of the total observable porosity (Figure 3a), which has been computed on the basis of tomography data, and weight loss (Figure 3b) defined experimentally, both for the full microstructures and also the filler and matrix sub-volumes. As might be expected, a higher porosity leads to greater $D_{0}$; this value tends to 3 for the full microstructures. The Hausdorff dimension is quite different for filler and matrix; the filler particle $D_{0}$ does not exceed 2.5, whilst the matrix $D_{0}$ lies in the region of 2.6 to 3. It is notable that the data for virgin HYB and HPB graphites are only slightly different, although the virgin HPB data are affected by the poor discrimination of matrix porosity. The dependences of $D_{0}$ on the mean porosity of the oxidised HPB samples can be 
approximated by power functions for matrix and full microstructure, whilst $D_{0}$ appears approximately constant for the filler particles within the weight loss range of $10-70 \%$. Data points associated with virgin microstructures and the lowest weight loss of $8 \%$ lies separately from this. Looking at the dependence of $D_{0}$ on weight loss in Figure 3b, one can see that these data points do not support the general behaviour. The percolation threshold, which is $31 \%$ for the total porosity in a 3D simple cubic lattice such as represented by the tomography image stack, will occur around a weight loss value of $\sim 10 \%$, so this may represent a structural re-organisation that has led to the qualitatively different state of the microstructure. These points are also located separately from the general set in Figure 3c and Figure 3e.

Since elastic modulus is a monotonic function of the total porosity that is affected by pore geometry, we might expect a relationship between elastic modulus values and the Rényi dimensions. We have compared elastic modulus values of the full microstructures, which were experimentally measured in the weight loss range of 0-53\% by EDF Energy Generation, with the values of the information dimensions calculated for the same microstructures. Figure 3c shows a linear function that describes the relationship between Young's modulus and $D_{1}$. In order to explore this relationship for individual filler particles and matrix sub-volumes, a geometrical approach for Young's modulus $(E)$ estimation has been developed on the basis of the semi-empirical relationship [31]:

$$
\frac{E}{E_{0}}=(1-a P)^{n}
$$

where $E_{0}$ is the modulus for a pore-free microstructure, $a$ is the packing geometry factor, and $n$ is the pore geometry factor. Extrapolation of the data to a pore-free state finds $E_{0} \approx 24 \mathrm{GPa}$ for $\mathrm{HYB}$ and $E_{0} \approx 20 \mathrm{GPa}$ for HPB. In its original formulation, $n$ is a constant that is 2.14 for closed spherical pores and 4.12 for irregular interconnected pores [31]. We assume that $a=1$, and propose that $n$ may be computed on the basis of the pore shapes. This can be done in a cubic lattice with step-like boundaries as a ratio between the free surface area $A_{\text {surf }}$ of the cells contacting to the pores orthogonally to the direction of the applied load, and the total free surface area $A_{\text {total }}$ of the pores:

$$
n=\left(\frac{A_{\text {surf }}}{A_{\text {total }}}\right)^{-1 / 2}
$$


This computation shows an almost linear relationship between the information dimension and elastic modulus of the full microstructure of both graphites and also sub-volumes of filler and matrix (Figure $3 d$ ). The lowest value of $E / E_{0}$, which corresponds to the highly oxidised microstructures, is associated with the highest values of $D_{1}$. This shows that as the graphite microstructure degrades with the radiolytic oxidation, this process increases the structural entropy of the system. There is also a difference between absolute values of the information dimension for filler and matrix for both graphite grades. The filler's porous structure demonstrates lower entropy, whilst the $D_{1}$ values of the matrix are close to the values for the full microstructure. Low entropy corresponds to a more organised structure with a certain configuration of a higher symmetry, which is quite stable during its evolution. Therefore, we can expect that the filler part of the microstructure will behave differently from the matrix under external action such as oxidation or mechanical loading. This is confirmed by our recent investigations of Gilsocarbon graphite [32, 33].

The correlation dimension has been also computed and is associated with the maximum equivalent diameter of the pores. The maximum pore size is difficult to estimate for the full microstructure due to the highly developed porous structure. More than $90 \%$ of all observable pores can be interconnected, thus representing a single pore; this reaches $99 \%$ for the highly oxidised graphite microstructures. The full microstructures have therefore not been analysed. The maximum pore sizes calculated for the selected sub-volumes are also limited by the total sizes of the extracted filler and matrix regions. This is observable for the HYB matrix, which has size $1 \mathrm{~mm}^{3}$ against $0.5^{3} \mathrm{~mm}^{3}$ of HPB matrix (Figure 3e) and, therefore, the maximum equivalent diameters are significantly larger. The dependence of $D_{2}$ on the maximum diameter presents a linear relationship for the HPB samples excluding the region of $0-10 \%$ of weight loss for the filler (Figure 3e). It should be noted that the results in this work have been obtained for the length scale of $>2.5 \mu \mathrm{m}$, due to the resolution of the tomographs, and an analysis of fractal parameters in higher resolution data, for instance, would not necessarily provide identical parameters.. 


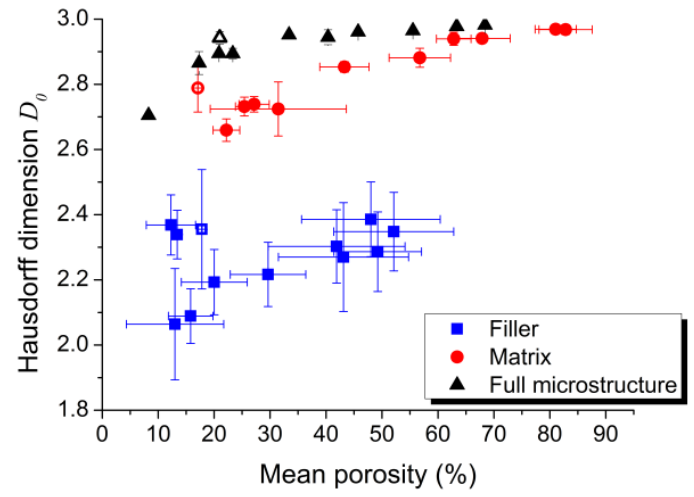

a)

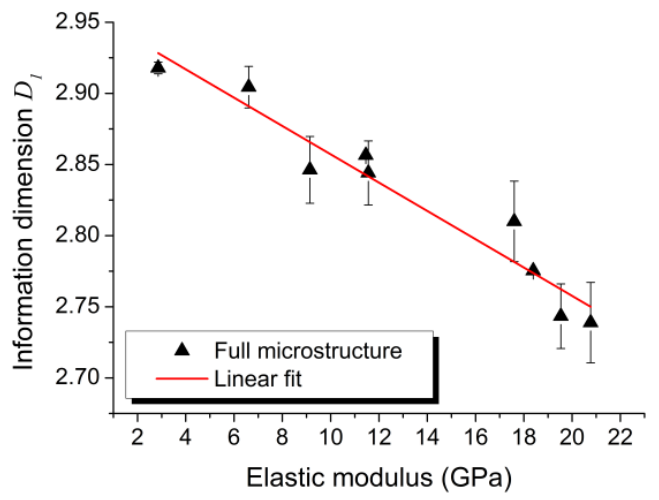

c)

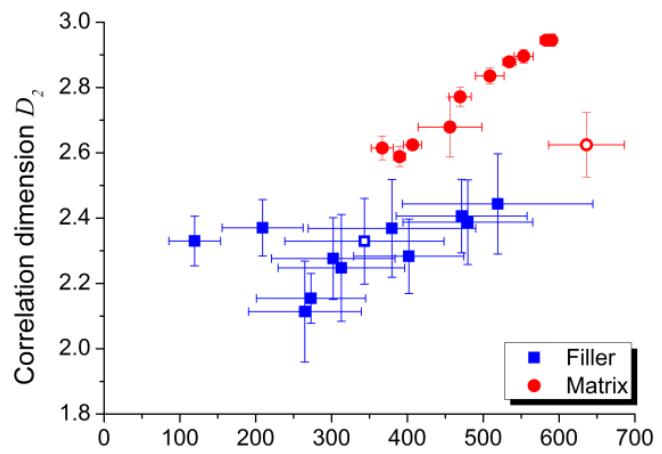

e)

Maximum equivalent diameter $(\mu \mathrm{m})$

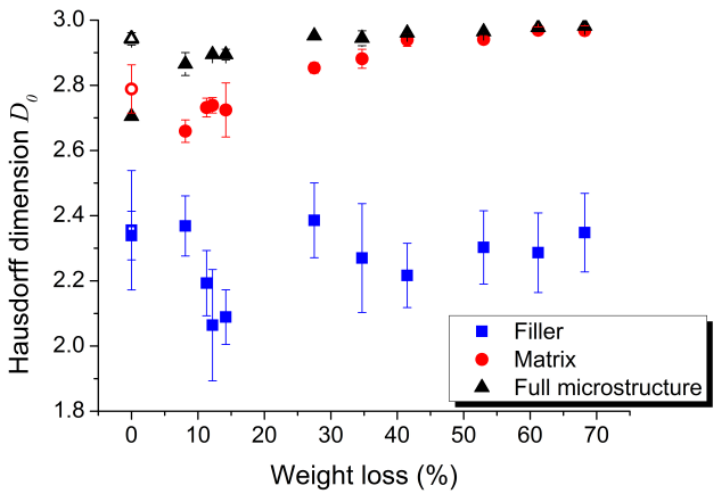

b)

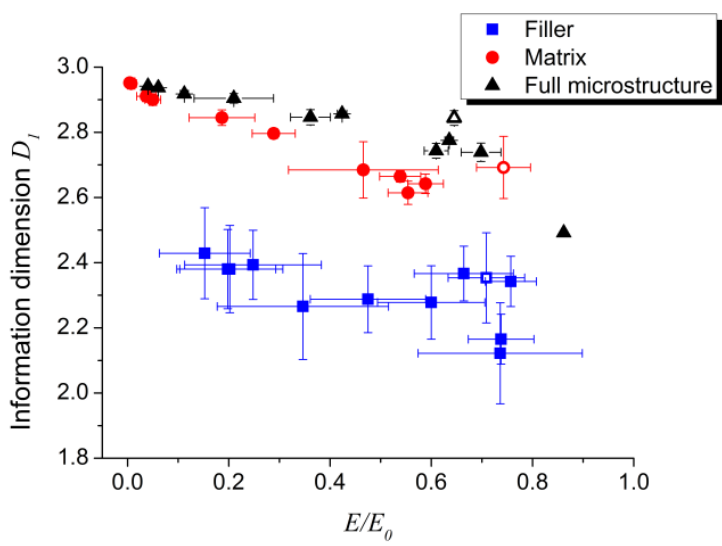

d)

Figure 3 - Behaviour of the fractal parameters for virgin and oxidised Gilsocarbon graphite. HPB graphite is shown by solid points, HYB graphite is represented by open points. 


\subsection{Multifractal-based algorithm for filler particle segmentation}

It is notable in Figure 3 that none of the Rényi dimensions of the filler particles exceed 2.5 in all cases for both graphite grades, and they are consistently lower than the matrix' values. Hence, on the basis of the observed multifractal features of the Gilsocarbon graphite microstructure, we have developed an algorithm for filler particle segmentation in threedimensional datasets.

The algorithm is applied to a 3D microstructure of Gilsocarbon graphite. It can be easily parallelised for greater efficiency and large datasets. The input data of the algorithm are a binary microstructure, a searching cubic window size $(w)$ and a percentage of window overlapping $(v)$ in the search. Any of the Rényi dimensions might be taken as a criterion of separation between filler and matrix structures. Here we have calculated the Hausdorff dimension. The parameter $w$ must be selected, relative to the data resolution, so that several sub-divisions of the cubic searching window would be possible in order to determine the scaling curve (e.g. as in Figure 2). If the obtained value of the Hausdorff dimension is lower than 2.5 , the region defined by the window is considered as a part of a filler particle.

The size of the searching window has an effect on the final result; the main positions of the particles can be captured readily, but optimisation is required for better discrimination. This is illustrated in Figure 4, which shows that a too small size of the window leads to greater noise in the output, whilst a too large window size loses some features of the microstructure. The window size parameter correlates with the average filler particle size, and so will be different for graphite grades with different filler particle size distributions. The overlapping parameter, $v$, defines the number of the voxels used for the shift step of the searching window; a higher value leads to smoother boundaries and internal continuity of the detected filler particles. 


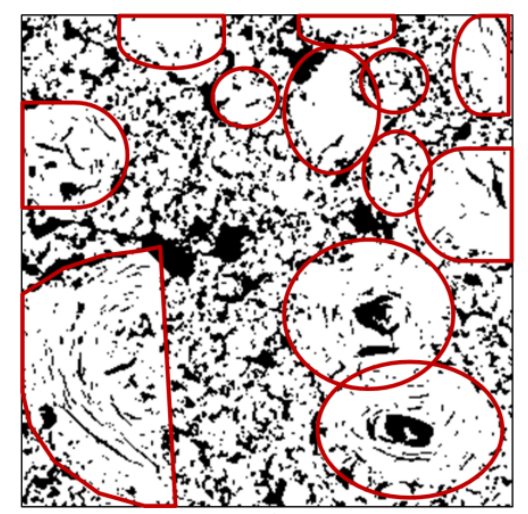

a)

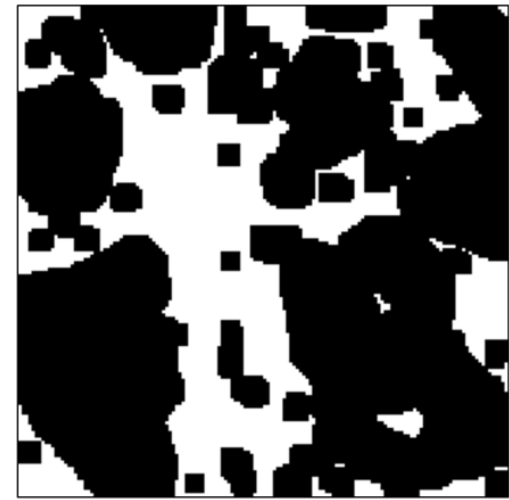

d)

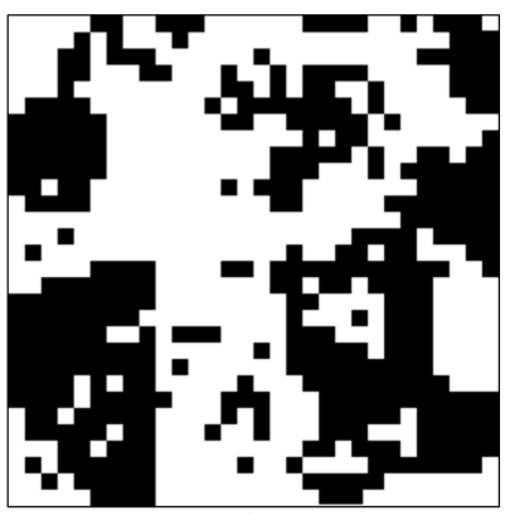

b)

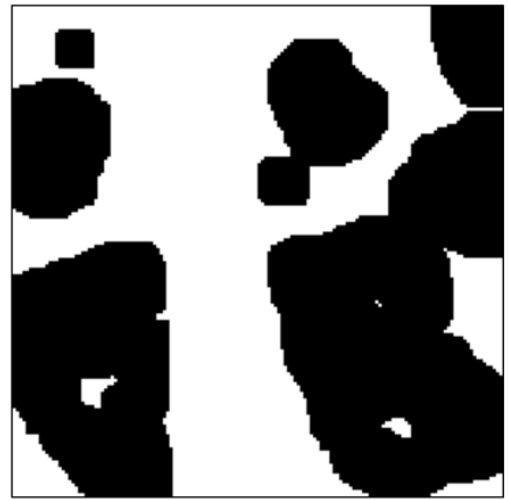

e)

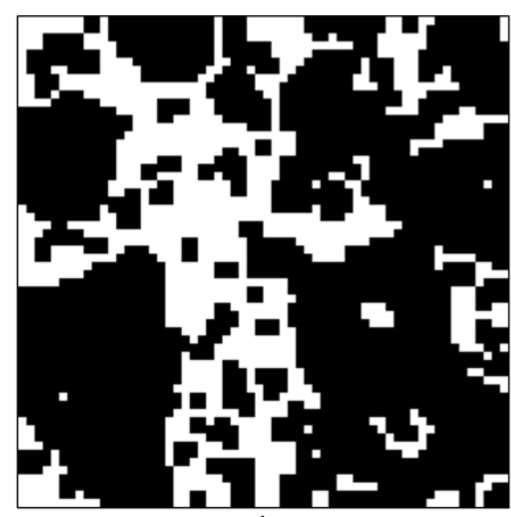

c)

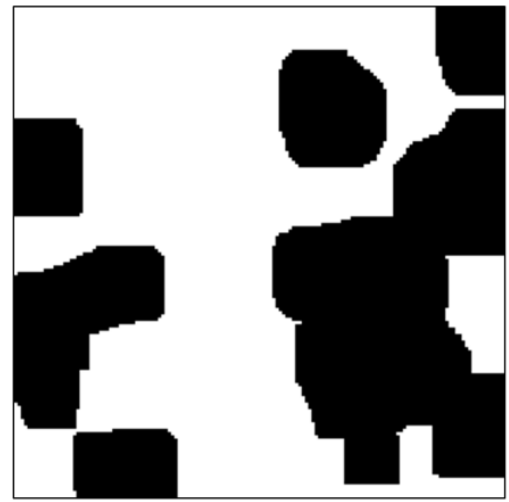

f)

Figure 4 - 2D binary image slice from a 3D HYB Gilsocarbon microstructure with visually defined filler particles (a) and results of the filler segmentation algorithm with different sizes of 3D searching window ( $w$ ) and percent of overlapping (v): b) $w=100 \mu \mathrm{m}, v=0$; c) $w=100$ $\mu \mathrm{m}, v=50 \%$; d) $w=120 \mu \mathrm{m}, v=83 \%$; e) $w=200 \mu \mathrm{m}, v=90 \%$; f) $w=300 \mu \mathrm{m}, v=93 \%$. The total size of the microstructure is $3 \times 3 \times 3 \mathrm{~mm}$. The optimum parameters are chosen to be $200 \mu \mathrm{m}$ and $90 \%$ (e).

As a demonstration, we have applied the developed algorithm for filler particle segmentation to a HYB Gilsocarbon microstructure, in which a crack has been initiated at a blunt notch. The development of the crack was studied by synchrotron X-ray tomography and digital volume correlation, and further details of the experiment can be found in [34]. Segmentation of the tomographs to detect cracks in graphite is difficult due to the high porosity of the microstructure, so digital volume correlation (DVC) is used to detect the crack, as DVC is sensitive to the opening displacements of the crack [35, 36]. In this case, the output is a maximum normal strain field, from the gradients of the displacement field, which has a resolution of $57.6 \mu \mathrm{m} /$ voxel. The following parameters have been used for the microstructure segmentation algorithm: $w=200 \mu \mathrm{m}, v=90 \%$, applied to the tomography dataset that had a size of 5 x 5 x $1.3 \mathrm{~mm}$ with a resolution of $9 \mu \mathrm{m} /$ voxel. 
The crack has a complex shape, which is difficult to observe in the tomograph (Figure 5a and b), but it can be segmented with the use of maximum normal strain data (Figure 5c). Visual comparison of its path with the filler particles, which were segmented by the multi-fractal analysis, indicates that the crack mostly propagated between the particles (Figure $5 \mathrm{~d}$ and e). This is confirmed quantitatively by calculation of the fraction of filler-occupied voxels that are coincident with the voxels of the strain-segmented crack; only $12 \%$ of the voxels recognised as the crack are coincident with filler, while the remaining $88 \%$ of the crack is located in the Gilsocarbon matrix. The proportions of filler and matrix defined in the total volume by multi-fractal segmentation are both $\sim 50 \%$.

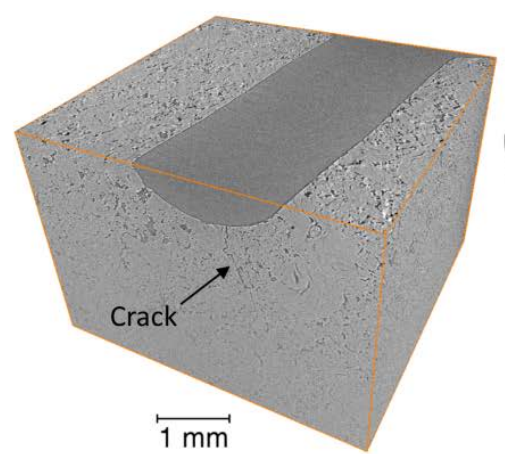

a)

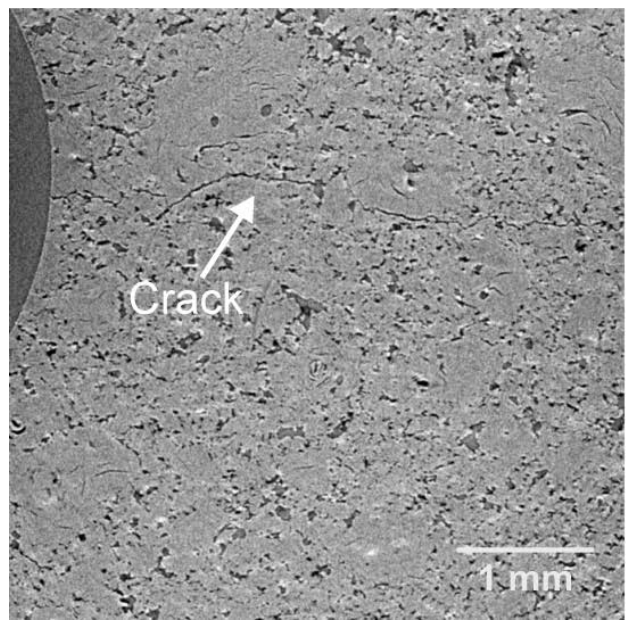

b)

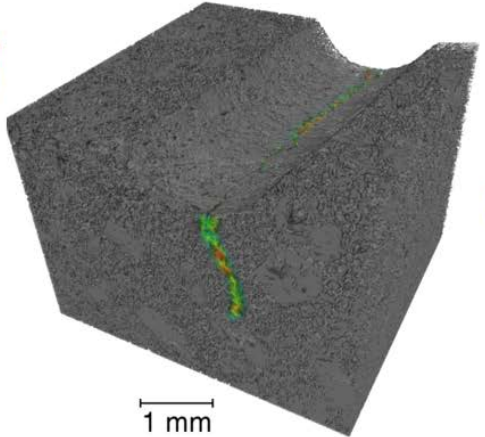

c)

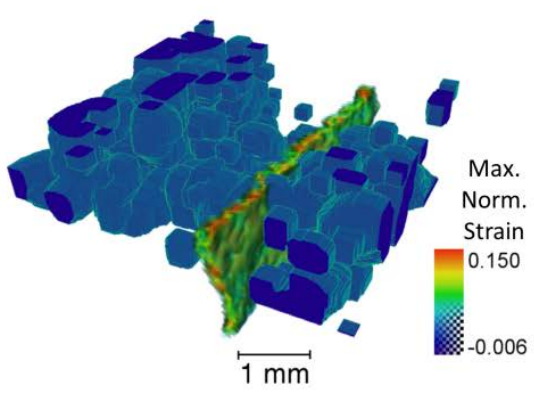

e)
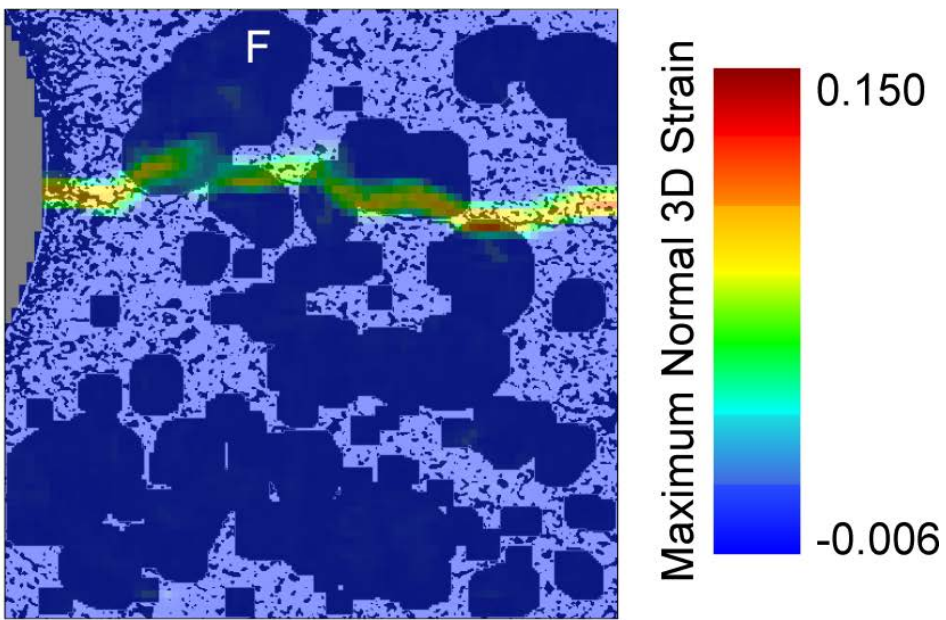

Figure 5 - 3D and 2D visualisations of a crack and microstructure in virgin HYB Gilsocarbon: a) reconstructed 3D X-Ray tomography dataset; b) X-Ray tomography 2D slice; c) $3 \mathrm{D}$ visualisation of the crack, segmented by maximum normal strain, in the microstructure, 
d) binary microstructure 2D image of the segmented filler particle (F) structure and the strain field; e) 3D visualisation of the segmented filler particles and crack.

This technique for the filler particle segmentation in Gilsocarbon graphite has potential application in the quantitative 3D characterisation of microstructures, for instance to support investigations of the effects of microstructure variations on mechanical properties [37-39] of the small specimens, which may control data scatter. Data on microstructure variations within manufactured components, which could be measured in quite large datasets using this method, could also provide insights into the variability of their performance [40-42]. The technique may be transferred to other materials, such as ceramic composites [43-46], that contain different pore structures in order to perform a segmentation of their individual components for modelling.

\section{Conclusions}

Multifractal properties of virgin and radiolytically oxidised Gilsocarbon microstructure have been revealed within the length scale of $>2.5 \mu \mathrm{m}$. It has been shown that the filler particles have Hausdorff dimension $D_{0}$ less than 2.5, whilst $D_{0}$ is higher than 2.5 for matrix regions and full microstructure. A linear relationship between the information dimension and elastic modulus of Gilsocarbon graphite is obtained. The correlation dimension that is associated with the pore sizes may be estimated on the basis of the available tomography data. A fractalbased algorithm for filler particle segmentation within Gilsocarbon microstructures has been developed. Application of the algorithm to the microstructure with the crack is encouraging; it confirms that a three-dimensional crack propagates predominantly within the matrix of virgin Gilsocarbon.

\section{Acknowledgments}

T.J.M. gratefully acknowledges the support of Oxford Martin School, and Ye.V. acknowledges the support of EDF Energy Generation (GRA/GNSR 6061). Mr M. Jordan is acknowledged for assisting in tomography data recording at the Diamond Light Source (Experiment EE8519) and provision of the DVC data. The views expressed in this paper are those of the authors, and not necessarily those of the project sponsors.

\section{References}

[1] B. Mandelbrot, The fractal geometry of nature, W.H. Freeman1982. 
[2] J.G. Evertsz, B.B. Mandelbrot, Multifractal measures, in: H.O. Peitgen, H. Jurgens, D. Saupe (Eds.), Chaos and Fractals: New frontiers of Science, Springer-Verlag New York1992, pp. 921-954.

[3] M.S. Jouini, S.Vega, E.A. Mokhtar, Multiscale Characterization of Pore Spaces Using Multifractals Analysis of Scanning Electronic Microscopy Images of Carbonates, Nonlin Processes Geophys 18 (2011) 941-953.

[4] A. Dathe, M. Thullner, The relationship between fractal properties of solid matrix and pore space in porous media, Geoderma 129 (2005) 279-290.

[5] J. Muller, Characterization of pore space in chalk by multifractal analysis, J Hydrol 187 (1996) 215-222.

[6] A. Giri, S. Tarafdar, P.Gouze, T. Dutta, Multifractal analysis of the pore space of real and simulated sedimentary rocks, Geophys J Int 200 (2015) 1106-1115.

[7] A.N. Khlyupin, O.Y. Dinariev, Fractal Analysis of the 3D Microstructure of Porous Materials, Technical Physics 60(6) (2015) 805-810.

[8] Y. Gao, J. Jiang, G.D. Schutter, G. Ye, W. Sun, Fractal and multifractal analysis on pore structure in cement paste, Construction and Building Materials 69 (2014) 253-261.

[9] X. Shanhua, R. Songbo, W. Youde, Three-Dimensional Surface Parameters and MultiFractal Spectrum of Corroded Steel, PLoS ONE 10(6) (2015) 1-15.

[10] U. Cikalova, M. Kroening, J. Shreiber, Y. Vertyagina, Evaluation of Al-specimen fatigue using a “smart sensor”, Physical Mesomechanics 14(5-6) (2012) 308-315.

[11] P.V. Kuznetsov, V.E. Panin, J. Shreiber, Fractal dimension as a characteristic of deformation stages of austenite stainless steel under tensile load, Theoretical and Applied Fracture Mechanics 35 (2001) 171-177.

[12] A.S. Balankin, A.D. Izotov, V.U. Novikov, Multifractal Analysis of Relations between Structural and Mechanical Parameters of Polymer-Matrix Composites, Inorganic materials 35(10) (1999) 1047-1053.

[13] A. Ebrahimkhanlou, A. Farhidzadeh, S. Salamone, Multifractal analysis of crack patterns in reinforced concrete shear walls, Structural Health Monitoring (2016).

[14] M.P. Dubuisson, R.C. Dubes, Efficacy of fractal features in segmenting images of natural textures, Pattern Recognition Letters 15 (1994) 419-431.

[15] J.P. Bonal, A. Kohyama, J. Van Der Laan, L.L. Snead, Graphite, ceramics, and ceramic composites for high-temperature nuclear power systems, MRS Bulletin 34(1) (2009) 28-34. [16] B.T. Kelly, Graphite - the most fascinating nuclear material, Carbon 20(1) (1982) 3-11. 
[17] L. Babout, P.M. Mummery, T.J. Marrow, A. Tzelepi, P.J. Withers, The effect of thermal oxidation on polycrystalline graphite studied by X-ray tomography, Carbon 43(4) (2005) 765-774.

[18] H. Imai, K. Fujii, T. Kurosawa, S. Nomura, Changes in Young’s Modulus and Electrical Conductivity of Nuclear Grade Graphites Oxidized with Air, J Nucl Mat 118 (1983) 294-302. [19] I.M. Pickup, B. McEnaney, R.G. Cooke, Fracture Processes in Graphite and the Effects of Oxidation, Carbon 24(5) (1986) 535-543.

[20] Q. Liu, Y.F. Hu, Q.L. Wang, Researches on Fractal Features of Graphite Porous Materials, Advanced Materials Research 507 (2012) 25-29.

[21] Q.L. Wang, M.He, Y.Q. He, J. Han, Fractal characteristics of the microstructure for porous graphite, Materials Research Innovations 19:sup1 (2015) S1-459-S1-464.

[22] Z. Zhou, W.G. Bouwman, H. Schut, S. Desert, J. Jestin, S. Hartmann, C. Pappas, From Nanopores to Macropores: Fractal Morphology of Graphite, Carbon 96 (2016) 541-547.

[23] M. Joyce, B. Davies, M.R. Bradford, Ongoing development of models to predict AGR core component condition, in: P.E.J. Flewitt, A.J. Wickham (Eds.) The 4th EDF Energy Nuclear Graphite Symposium, EMAS Publishing, Nottingham, 2015, pp. 195-212.

[24] G.M. Laudone, C.M. Gribble, G.P. Matthews, Characterisation of the porous structure of Gilsocarbon graphite using pycnometry, cyclic porosimetry and void-network modeling, Carbon 73 (2014) 61-70.

[25] H. Gould, J. Tobochnik, More on Fractals and Chaos: Multifractals, Computers in Physics 4(2) (1990) 202.

[26] C.E. Shannon, The mathematical theory of communication, The Bell System Technical Journal 27 (1948) 379-423.

[27] S.G. Karstina, K.S. Baktybekov, Y. Vertyagina, Analysis of the Luminescence Decay on the SiO2 Surface at Different Temperatures within the Multifractal Formalism, Russian Physics Journal 48(6) (2005) 553-558.

[28] Y. Vertyagina, Research of Open Systems Evolution by the Method of Multifractal Analysis, Chaos and Structures in Nonlinear Systems. Theory and Experiment, Astana, 2006. [29] J. Shreiber, U. Cikalova, Y. Vertyagina, Use of the fractal nature of spatial and temporal response behavior for materials damage characterization, 6th International Conference on Low Cycle Fatigue, Berlin, 2008, pp. 655-660.

[30] C.A. Schneider, W.S. Rasband, K.W. Eliceiri, NIH Image to ImageJ: 25 years of image analysis, Nat Meth 9(7) (2012) 671-675. 
[31] K.K. Phani, S.K. Niyogi, Young’s modulus of porous brittle solids, J Mater Sci 22 (1987) 257-263.

[32] T.J. Marrow, D. Liu, S.M. Barhli, L. Saucedo-Mora, Y. Vertyagina, D.M. Collins, In situ measurement of the strains within a mechanically loaded polygranular graphite, Carbon 96 (2016) 285-302.

[33] Y. Vertyagina, T.J. Marrow, 3D Cellular Automata fracture model for porous graphite microstructures, 23rd Conference on Structural Mechanics in Reactor Technology, Manchester, 2015.

[34] T.J. Marrow, M.S.L. Jordan, Y. Vertyagina, Towards a notch-sensitivity strength test for irradiated nuclear graphite structural integrity, in: P.E.J. Flewitt, A.J. Wickham (Eds.) The 4th EDF Energy Nuclear Graphite Symposium. Engineering Challenges Associated with the Life of Graphite Reactor Cores, EMAS Publishing, Nottingham, 2015, pp. 247-259.

[35] M. Mostafavi, N. Baimpas, E. Tarleton, R.C. Atwood, S.A. McDonald, A.M. Korsunsky, T.J. Marrow, Three-dimensional crack observation, quantification and simulation in a quasi-brittle material, Acta Mater 61(16) (2013) 6276-6289.

[36] Y. Vertyagina, M. Mostafavi, C. Reinhard, R. Atwood, T.J. Marrow, In situ quantitative three-dimensional characterisation of sub-indentation cracking in polycrystalline alumina, Journal of the European Ceramic Society 34(12) (2014) 3127-3132.

[37] T.D. Burchell, A Microstructurally Based Fracture Model for Nuclear Graphite, Carbon 34(3) (1996) 297-316.

[38] N. Nemeth, A. Walker, E. Baker, P. Murthy, R. Bratton, Large-scale Weibull analysis of H-451 nuclear-grade graphite rupture strength, Carbon 58 (2013) 208-225.

[39] M.P. Hindley, D.C. Blaine, A.A. Groenwold, T.H. Becker, Failure prediction of full-size reactor components from tensile specimen data on NBG-18 nuclear graphite, Nuclear Engineering and Design 284 (2015) 1-9.

[40] T. Arai, S. Sato, T. Oku, H. Schiffers, W. Delle, Assessment of Heterogeneity and Anisotropy of IG-110 Graphite for Nuclear Components, Journal of Nuclear Science and Technology 28(8) (1991) 713-720.

[41] S. Yu, X. Fang, H. Wang, C. Li, Failure probability study of HTR graphite component using microstructure-based model, Nuclear Engineering and Design 253 (2012) 192-199.

[42] S.-H. Chi, Specimen size effects on the compressive strength and Weibull modulus of nuclear graphite of different coke particle size: IG-110 and NBG-18, Journal of Nuclear Materials 436(1-3) (2013) 185-190. 
[43] Y. Nikishkov, L. Airoldi, A. Makeev, Measurement of voids in composites by X-ray Computed Tomography, Composites Science and Technology 89 (2013) 89-97.

[44] G.W. Melenka, E. Lepp, B.K.O. Cheung, J.P. Carey, Micro-computed tomography analysis of tubular braided composites, Composite Structures 131 (2015) 384-396.

[45] C. Zou, T.J. Marrow, C. Reinhard, B. Li, C. Zhang, S. Wang, Porosity characterization of fiber-reinforced ceramic matrix composite using synchrotron X-ray computed tomography, Journal of Instrumentation 11(03) (2016) C03052.

[46] L. Saucedo-Mora, T.J. Marrow, Multi-scale damage modelling in a ceramic matrix composite using a finite-element microstructure meshfree methodology, Philosophical Transactions of the Royal Society of London A: Mathematical, Physical and Engineering Sciences 374(2071) (2016). 